\title{
COVID-19: exposición perinatal. Perspectivas
} COVID-19: Perinatal exposure. Perspectives

Después de 15 meses del inicio de la pandemia mundial de coronavirus ¿qué sabemos sobre la infección?

Desde el punto de vista de la ciencia, como nunca antes, los investigadores de todo el mundo se han abocado al estudio de una patología, de su cura y/o prevención. Prueba de ello, son las numerosas vacunas aprobadas en emergencia que se están distribuyendo y aplicando (aunque lentamente) a la población mundial, mientras siguen los estudios para concluir los resultados y se desarrollan nuevas vacunas.

Desde el punto de vista de la salud pública, en la mayor crisis mundial jamás vivida se han puesto de manifiesto muchas dificultades y deficiencias en las estructuras y recursos (edilicias, técnicas, humanas entre otras) de los sistemas sanitarios de muchos países, especialmente en las regiones y comunidades más vulnerables. ${ }^{1}$

Al poco tiempo de conocerse la rápida expansión del COVID-19, en Archivos comenzamos a publicar guías de manejo, actualizaciones y artículos que dan cuenta del impacto de la pandemia en la salud psicofísica y social de la población pediátrica, así como también, sobre la preparación de los servicios y profesionales de la salud para afrontar el coronavirus al comienzo de la pandemia y sobre algunos resultados de la adaptación y los cambios producidos en la atención de niños, niñas y adolescentes.

Al comienzo de la pandemia se observó que los niños no eran la población blanco de la infección por SARS-CoV-2, en su mayoría cursaban infecciones leves y que las tasas de hospitalización por enfermedad grave, ingreso a ventilación mecánica y muerte eran bajas con respecto a otros grupos etarios o pacientes con factores de riesgo o comorbilidades.

Asimismo, los primeros datos publicados mostraban que las mujeres embarazadas (a diferencia de la pandemia por gripe A H1N1 en el año 2009) tampoco tenían mayor riesgo de contraer la infección y estar gravemente enfermas o morir a causa de ésta. Sobre los recién nacidos, hijos de madres SARS- CoV-2 positivas, los primeros estudios reportaban muy baja probabilidad de contraer la enfermedad in útero, al nacimiento o luego en el post parto.

Duran y col. en una revisión sistemática en abril del 2020 cuyo objetivo fue describir los resultados perinatales y neonatales en recién nacidos expuestos al SARS-CoV-2 reportaron que de 222 recién nacidos incluidos de estudios seleccionados, solo 13 fueron positivos y que la mayoría de los estudios informaron síntomas leves o nulos y ningún resultado perinatal adverso. En los estudios que usaron datos sobre sangre de cordón umbilical, placenta o líquido amniótico no se informaron resultados positivos y tampoco en aquellos que investigaron en leche materna. Los autores concluyeron que la evidencia era todavía escasa, que la transmisión vertical no se podía confirmar y no recomendaba separar al niño de su madre o suspender la lactancia materna. ${ }^{2}$

Por otra parte, otros autores mostraron que las mujeres embarazadas con un diagnóstico confirmado de COVID-19 tenían un mayor riesgo de parto prematuro y cesárea comparadas con embarazadas no infectadas, aunque vieron que el aumento de cesárea incluyó la indicación médica antes del trabajo de parto sin causa materna o fetal (iatrogénica). ${ }^{3}$

Llamativamente dos estudios realizados en Dinamarca ${ }^{4}$ y en Irland $a^{5}$ informaron que durante la cuarentena impuesta por sus gobiernos se observaron menores tasas de nacimientos prematuros o mortinatos que en años anteriores y no se pudo establecer la causa. ${ }^{4}$ El determinante se especula que podría ser el impacto socioambiental del confinamiento. ${ }^{5}$ Otra publicación, sin embargo informó que en un hospital del Reino Unido hubo una incidencia aumentada de mortinatos durante la pandemia (ninguno asociado a COVID-19) comparado con un período anterior prepandémico. Los autores infieren distintas causas (renuencia a ir al hospital, disminución de controles y estudios prenatales por escasez de personal, hipertensión materna subdiagnosticada por falta de controles, entre otras). ${ }^{6}$ Por el contrario, otros estudios en EE. UU. no encontraron diferencias en la tasas de mortinatos o partos prematuros durante el pico más alto de la pandemia o el confinamiento. ${ }^{7,8}$

Un informe reciente del CDC expone que "Las mujeres embarazadas tienen un mayor riesgo de sufrir COVID-19 grave que las mujeres no embarazadas, y que el COVID-19 se asocia con un mayor riesgo de parto prematuro". ${ }^{9}$

Todavía, poco se sabe sobre la vía de transmisión del virus de la madre al recién nacido. En un artículo de revisión recientemente publicado, Wong y col. concluyen que "la evidencia parece sugerir que la transmisión vertical intrauterina ocurre, pero raramente". De los estudios revisados observaron que, "aunque la mayoría de los recién nacidos de madres 
infectadas con SARS-CoV-2 no parecen adquirir la infección después del parto, se habían notificado casos de recién nacidos complicados con viremia y compromiso neurológico posterior. Todavía están bajo estudio los mecanismos de transmisión". En su revisión "tampoco encontraron informes de efectos teratogénicos del virus en los fetos". ${ }^{10}$

Algunos reportes de casos informan la presencia de anticuerpos en el recién nacido a las pocas horas del nacimiento lo que indica que existiría transmisión vertical. Asimismo surgen otras preguntas: ¿el momento de la gestación en el que se contrae la infección influye o modifica la evolución del binomio? o ¿es diferente la transmisión del virus o la evolución del neonato si la madre cursa una enfermedad moderada o grave? Schwartz y Morotti, en una comunicación resumen cuáles podrían ser los potenciales efectos del SARS-CoV-2 en la placenta y la fisiopatología de la infección materno-fetal así como también los posibles factores que aumenten el riesgo de infección placentaria con SARS-CoV-2 y que pueden favorecer la transmisión del virus al feto. ${ }^{11}$

Lo que sí se sabe es que las evidencias, escasas por ahora, muestran que los recién nacidos de madres con SARS-CoV-2 confirmado o sospechado, la mayoría de las veces no adquieren la infección o son asintomáticos y los sintomáticos en general presentan una evolución favorable. Esto apoya las recomendaciones nacionales e internacionales en favor de la no separación de la madre y el niño y fomentar la lactancia materna con medidas de cuidado apropiadas.

En un informe del Ministerio de Salud de la Provincia de Buenos Aires de la Dirección Provincial de Equidad de Género en Salud, Dirección de Maternidad y Salud Perinatal, de vigilancia epidemiológica activa de COVID-19 en gestantes y neonatos hasta el mes de vida, realizado desde el primer caso materno reportado en la semana epidemiológica 13 hasta la semana 39 del 2020, se observó que de 5520 gestantes con sospecha de infección SARS-CoV-2, 2283 (el 41 \%) fueron casos confirmados según definición ministerial actualizada. De ellas el 96,2 \% tuvo síntomas leves y el 2,1 \% (49) ingresó a UCI; de ellas 20 requirieron asistencia respiratoria mecánica y 9 pacientes (el 0,4\%) fallecieron. Sobre 161 casos de gestantes en seguimiento que concluyeron el embarazo un $25,5 \%$ tuvo un recién nacido pretérmino; aborto, muerte fetal y neonatal se produjeron en porcentajes bajos. En el período monitoreado, sobre 1009 neonatos seguidos hasta el mes de vida, 140 fueron positivos para SARS-CoV-2 (el 13,9\% antes del mes), de ellos 7 fallecieron (el $5 \%$ ). Las formas de presentación fueron en su mayoría fiebre y síntomas respiratorios. El mayor porcentaje de los recién nacidos fueron de término $(\mathrm{el} 74 \%)$ con resolución favorable en $72 \mathrm{~h}$.

A nivel nacional, por el momento, hay pocos datos sobre la incidencia de la infección por SARS-CoV-2 en embarazadas, la letalidad en las gestantes enfermas, la incidencia de la infección y la evolución en los recién nacidos.

La pandemia por coronavirus es un acontecimiento dinámico y en proceso. Se necesita seguir acumulando evidencia sobre la exposición perinatal de la infección, la fisiopatología de la enfermedad, la evolución y el seguimiento estrecho de la madre y su recién nacido.

\section{Dra. Adriana Aguilar Editora asistente}

http: / / dx.doi.org/10.5546/ aap.2021.146

Texto completo en inglés:

http:/ / dx.doi.org/10.5546/ aap.2021.eng.146

Cómo citar: Aguilar A. COVID-19: exposición perinatal. Perspectivas. Arch Argent Pediatr 2021;119(3):146-147.

\section{REFERENCIAS}

1. Benjamin GC. Asegurar la equidad en la salud durante la pandemia de COVID-19: el papel de la infraestructura de salud pública [Consulta: 4 de marzo de 2021] Disponible en: https: / / www.paho.org/journal / es / articulos / asegurar-equidad-salud-durante-pandemia-covid-19papel-infraestructura-salud-publica

2. Duran P, Berman S, NiermeyerS, Jaenisch T, et al. COVID-19 and newborn health: systematic review. Rev Panam Salud Publica. 2020;44:e54.

3. Yang R, Mei H, Zheng T, Fu Q, et al. Pregnant women with COVID-19 and risk of adverse birth outcomes and maternal-fetal vertical transmission: a population-based cohort study in Wuhan, China. BMC Med. 2020;18(1):330.

4. Hedermann G, Hedley PL, Bækvad-Hansen M, Hjalgrim $\mathrm{H}$, et al. Danish premature birth rates during the COVID-19 lockdown. Arch Dis Child Fetal Neonatal Ed 2021;106(1):93-5.

5. Philip RK, Purtill H, Reidy E, Daly M, et al. Unprecedented reduction in births of very low birthweight (VLBW) and extremely low birthweight (ELBW) infants during the COVID-19 lockdown in Ireland: a 'natural experiment' allowing analysis of data from the prior two decades. BMJ Global Health. 2020;5(9):e003075.

6. Khalil A, von Dadelszen P, Draycott T, Ugwumadu A, et al. Change in the incidence of stillbirth and preterm delivery during the COVID-19 pandemic. JAMA.2020;324(7):705-6.

7. Wood R, Sinnott C, Goldfarb I, Clapp M, et al. Preterm birth during the coronavirus disease 2019 (COVID-19) pandemic in a large hospital system in the United States. Obstet Gynecol. 2021;137(3):403-4.

8. Handley SC, Mullin AM, Elovitz MA, Gerson KD, et al. Changes in preterm birth phenotypes and stillbirth at 2 Philadelphia hospitals during the SARS-CoV-2 pandemic, March-June 2020. JAMA. 2021;325(1):87-9.

9. Investigación del impacto del COVID-19 durante el embarazo. Actualizado el 4 de feb. del 2021. CDC: 2021. [Consulta: 6 de marzo de 2021]. Disponible en: https:/ / espanol.cdc.gov/coronavirus/2019-ncov/cases-updates / special-populations / pregnancy-data-on-covid-19/ whatcdc-is-doing.html

10. Wong YP, Khong TY, Tan GC. The effects of COVID-19 on placenta and pregnancy: What do we know so far? Diagnostics (Basel). 2021;11(1):94.

11. SchwartzDA, MorottiD. Placental pathology of COVID-19 with and without fetal and neonatal infection: trophoblast necrosis and chronic histiocytic intervillositis as risk factors for transplacental transmission of SARS-CoV-2. Viruses. 2020;12(11):1308. 